\title{
Aortic sac enlargement after endovascular aneurysm repair: volume-related changes and the impact of intraluminal thrombus
}

\author{
Arminas Skrebunas ${ }^{1,2 A, B, C, D, E, F}$, Givi Lengvenis ${ }^{2 B}$, Inga Urte Builyte ${ }^{1 E, F}$, Ruta Zulpaite ${ }^{1 B, E, F}$, Rytis Bliudzius ${ }^{3 B}$, \\ Tomas Baltrunas ${ }^{1,2 E}$, Nerijus Misonis ${ }^{1,2 E}$, Germanas Marinskis ${ }^{1,2 A, E}$ \\ 'Clinic of Cardiovascular Diseases, Faculty of Medicine, Vilnius University, Vilnius, Lithuania \\ ${ }^{2}$ Centre of Cardiology and Angiology, Vilnius University Hospital Santaros Klinikos, Vilnius, Lithuania \\ ${ }^{3}$ Centre of Radiology and Nuclear Medicine, Vilnius University Hospital Santaros Klinikos, Vilnius, Lithuania
}

\section{Abstract}

Purpose: Abdominal aortic aneurysm (AAA) growth after endovascular aneurysm repair (EVAR) is still unpredictable. The issue of optimal frequency of computed tomography angiography for surveillance and its measurement method accuracy remain unclear. We aimed to assess the value of abdominal aneurysm sac volume measurement for detecting expansions and the association of preprocedural intraluminal thrombus (ILT) volume with aneurysm sac growth following EVAR.

Material and methods: A total of 107 patients underwent elective EVAR. Inclusion criteria provided a cohort of 39 patients. Changes of postoperative maximum aneurysm sac diameter and AAA volume were calculated. Volumetric AAA changes and demographic data of the cases with clinically irrelevant AAA diameter enlargement were evaluated. Preoperative ILT volumes were collected. ILT and AAA sac volume ratio was calculated. Statistical data analysis was performed using standard methods.

Results: The mean changes of maximum AAA diameter and volume in percentage after EVAR were $-5.08 \pm 8.20 \mathrm{~mm}$ and $-13.39 \pm 23.32 \%$, respectively. A moderate positive linear correlation between those changes was found $\left(R^{2}=0.731 ; p<0.0001\right)$. The mean relative AAA volume increase in cases without clinically relevant diameter enlargement was $11.50 \pm 8.27 \%$. The means of ILT and AAA sac ratios were $0.59 \pm 0.17$ and $0.52 \pm 1.8$ in growing AAA sac and in stable or shrinking AAA sac groups, respectively $(p=0.308)$.

Conclusions: Volumetric AAA measurement may be useful as an additional method to diameter measurement after EVAR to identify clinically relevant sac growth. Preoperative volume of ILT may not significantly affect the growth rate of AAA after EVAR.

Key words: abdominal aortic aneurysm, intraluminal thrombus, EVAR, follow-up, AAA diameter, AAA volume.

\section{Introduction}

Endovascular aneurysm repair (EVAR) is the most frequently chosen treatment method for abdominal aortic aneurysm (AAA). Although it is a well-established, minimally invasive procedure with good perioperative results and short hospital stay, EVAR is associated with a three to four times higher rate of reintervention than open surgery, which leads to late aneurysm-related mortality [1]. Because exclusion of the aneurysm sac is not always definite in endovascular approach, a small but significant risk of AAA rupture remains, mainly due to endoleaks or stent graft migration [2,3]. Post-EVAR aneurysm sac growth without visible endoleaks may be the result of endotension. A study by Koole et al. revealed that the risk of AAA rupture in patients with sac enlargement of $8 \mathrm{~mm}$ but

Correspondence address:

Arminas Skrebunas, Clinic of Cardiovascular Diseases, Faculty of Medicine, Vilnius University, Vilnius, Lithuania, e-mail: arminas.skrebunas@gmail.com

Authors' contribution:

A Study design · B Data collection · C Statistical analysis · D Data interpretation · E Manuscript preparation · F Literature search · G Funds collection 
without endoleak is $<1 \%$ during the first four years, but later the rates increase to between $7.5 \%$ and $13.6 \%$ [4].

Whereas aneurysm growth remains unpredictable after EVAR, regular surveillance imaging including computed tomography angiography (CTA) is necessary. Several guidelines for surveillance following EVAR have been established; however, the issue of optimal frequency of CTA is still under discussion. It has been noticed that patients with normal CTA at one month after EVAR do not develop complications requiring reintervention for another three years, which suggests the possibility of decreasing the frequency of imaging in such cases [5]. Several predictive models of post-EVAR complications have been proposed, which would allow the adjustment of surveillance, according to individual risk [6-8]. Moreover, a few recent studies have suggested that aneurysm sac volume measurement is a more accurate method to identify patients requiring vigilant follow-up or reintervention than conventional assessment of maximum aneurysm diameter $[9,10]$. Nevertheless, not all factors influencing aneurysm sac behaviour and outcomes after endovascular procedure are clear. One of the widely discussed AAA growth risk factors is intraluminal thrombus (ILT). Results of different studies are controversial [11-13], but it is clear that ILT is a biochemically active structure, which has both inflammatory and mechanical effects on the aneurysm wall. Therefore, more data are necessary to decide what the summative effect actually is.

This study was designed to investigate the value of aneurysm sac volume measurement in addition to diameter measurements based on CTA after EVAR in the prediction of aneurysm progression. The secondary outcomes included determining whether there were any associations of preprocedural intraluminal thrombus volume with the aneurysm sac growth following EVAR.

\section{Material and methods}

The study was approved by the Local Ethics Committee (registered 13/12/2016 reg. 158200-16-877-386) and was conducted according to the principles of the Helsinki Declaration.

This is a longitudinal observational cohort study evaluating aneurysm parametric outcomes after EVAR. Patients undergoing elective EVAR between January 2007 and September 2017 in the university hospital were included in the study. A total of 107 patients underwent EVAR during that period. Inclusion criteria were: written, informed consent; age over 50 years; preoperative CTA scan of the entire aorta; at least two CTA scans after EVAR during the first two years of follow-up; and no endoleak or type 5 endoleak ("endotension"). These criteria provided a cohort of 39 patients for further analysis.

One hundred and seventeen CTA scans (39 preoperative and 78 postoperative) were evaluated by two independent radiologists. The data on preoperative AAA volume and intrasac thrombus volume were collected. ILT and AAA sac ratio was calculated. Postoperative maximum aneurysm sac diameter and volume were collected. The change of maximum aneurysm sac diameter and relative AAA volume change between the first and the last examinations postoperatively were evaluated. According to the postoperative volumetric AAA sac changes, all patients were divided into two groups: growing AAA sac group $(n=12)$ and stable or shrinking AAA sac group ( $n$ =27). Clinically relevant AAA sac expansion after EVAR remains unclear for patients with no endoleaks after EVAR. According to Society for Vascular Surgery guidelines, it was defined as an increase of at least $5 \mathrm{~mm}$ for all AAA sac expansion cases [14]. However, up to $2 \mathrm{~mm}$ error in measuring the diameter may occur using non-electrocardiographic (ECG)-gated CTA [15]. Non-ECG-gated CTA scans are performed at our institution. So, according to the literature, clinically relevant AAA sac expansion was defined as an increase of more than $5 \mathrm{~mm}$ in maximum diameter in this study. The volumetric AAA changes and demographic data of the cases with clinically irrelevant AAA diameter enlargement were evaluated.

All CT scans were performed using helical CT scanners GE (General Electric Healthcare, Waukesha, Wisconsin, United States) LightSpeed VCT (until 2012) and GE Discovery CT750 HD (since 2013) under a set acquisition protocol. Contrast-enhanced images were obtained after injecting 70-120 $\mathrm{ml}$ of non-ionic intravenous contrast matter. Images were initially reconstructed in axial planes with a slice thickness of $0.625 \mathrm{~mm}$.

CT images were transferred to Vitrea (Vital Images, Inc., Minnetonka, Minnesota, United States) picture archiving and communication system (PACS) archive for initial assessment of aneurysm sack and stent graft location, which was performed using Vital Vitrea 6.7.2 software. For measurements of aneurysm length, maximum aneurysm diameter, aneurysm volume, lumen and thrombus volume the Vitrea Advanced aorta "stent graft planning" protocol was used. Automated 3D segmentation by the software was performed, followed by manual adjustments to the centreline and outlines of the aneurysm and lumen in each slice where it was considered inaccurate. On average, the processing and evaluation of a single examination took $28.9 \pm 2.3 \mathrm{~min}$ and $30.1 \pm$ $0.8 \mathrm{~min}$ for the two radiologists accordingly. Maximum aneurysm diameter measurements were taken in orthogonal images perpendicular to the axis of aorta, measuring outer wall to outer wall (Figure 1). All measurements were recorded in millimetres (to the nearest $0.1 \mathrm{~mm}$ ). Volumetric measurements included the portion of the abdominal aorta, aneurysm, and iliac arteries covered by stentgraft (Figure 2). The software computed the volume of aneurysm (including wall of aorta), lumen, and thrombus according to the outlines in millilitres $(\mathrm{ml})$. The thrombus volume was calculated by subtracting the luminal volume from the AAA volume. 


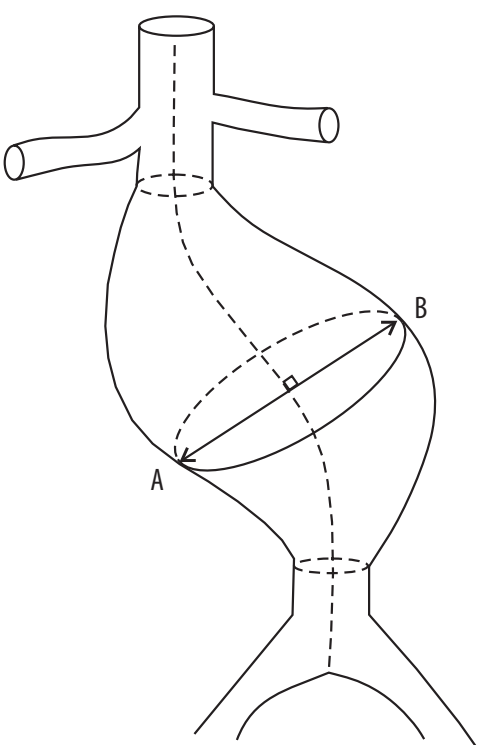

Figure 1. Maximum aneurysm diameter measurements ( $A B$ ) were taken in orthogonal images

Statistical data analysis was performed using IBM Statistical Package for the Social Sciences (SPSS) Version 25.0 (Armonk, NY, USA, IBM Corp.).

Descriptive statistics of patients' baseline characteristics and the interval between two CTA scans were calculated. Bland-Altman analysis was performed to determine the correlation between measurements done by two independent radiologists. The bias was calculated as the average difference between their results. Parametric data were presented in terms of the mean value and standard deviation (SD). All nominal data were presented as an absolute number and percentage prevalence in the study population. The Shapiro-Wilk test for normality was used to examine continuous variables. A Pearson's correlation was used to examine the linear relationship between two variables. AAA sac diametric and volumetric changes were compared using paired $t$-test. One-way analysis of variance (ANOVA) was used to determine whether there were any statistically significant differences between parameters in groups. All $p$-values were two-sided, and a $p$-value of 0.05 was considered statistically significant.

Table 1. Baseline characteristics of patients

\begin{tabular}{|l|c|}
\hline Characteristic & $N=39$ \\
\hline Age (years) & $70.3 \pm 8.2$ \\
\hline Gender (\%) & \\
\hline Female & 10.3 \\
\hline Male & 89.7 \\
\hline Cardiovascular risk factors (\%) & \\
\hline Smoker & 23.1 \\
\hline Hypertension & 87.2 \\
\hline Diabetes & 10.3 \\
\hline Chronic obstructive pulmonary disease & 15.4 \\
\hline
\end{tabular}

\section{Results}

\section{Baseline characteristics}

There were 107 patients initially included in the study, $14(13.1 \%)$ of whom were excluded because of endoleaks that developed during the surveillance period. The study also excluded 10 (9.3\%) patients who had had other non-endoleak-related complications such as limb occlusion or stent graft migration after EVAR. Forty-four (41.1\%) patients were excluded from the further analysis because of incomplete or no surveillance. Finally, 39 (36.5\%) patients were studied, comprising four (10.3\%) women and $35(89.7 \%)$ men; mean age $70.3 \pm 8.2$ years; age ranging between 51 and 85 years.

Baseline characteristics of the patients are shown in Table 1.

\section{Reproducibility}

The Bland-Altman plot was used to assess interobserver variability, finding no significant difference between the means of maximum aortic aneurysm diameter $(p>0.05)$, volume $(p>0.05)$, and ILT volume measurements $(p>0.05)$, the mean differences being $0.4 \pm 0.08 \mathrm{~mm}$, $2.1 \pm 0.67 \mathrm{ml}$, and $1.9 \pm 0.83 \mathrm{ml}$, respectively (Figure 3A-C).

\section{Abdominal aortic aneurysm sac parametric changes}

The mean follow-up time was $635.3 \pm 249.7$ days. The CT scans were not performed at the same time intervals for each patient because a follow-up algorithm had not been developed at that time. The first follow-up CT was performed after $61.1 \pm 109.9$ days, and the second $635.2 \pm$ 249.7 days after EVAR.

The mean change of maximum AAA diameter after EVAR was $-5.08 \pm 8.20 \mathrm{~mm}$. The increase was observed in $11(28.2 \%)$ cases with a mean of $3.35 \pm 2.53 \mathrm{~mm}$. It decreased in $28(71.8 \%)$ cases with a mean of $-8.39 \pm 7.21 \mathrm{~mm}$.

The mean change of AAA volume percentage after EVAR was $-13.39 \pm 23.32 \%$. It increased in $12(30.8 \%)$ cases and decreased in 27 (69.2\%) cases, with means of $13.34 \pm 15.25 \%$ and $-25.27 \pm 14.84 \%$, respectively.

The correlation between the maximum AAA diameter changes and the relative AAA volume changes after EVAR was determined. After performing regression analysis, a statistically significant and moderate positive linear correlation was found $\left(R^{2}=0.731 ; p<0.0001\right)$ (Figure 4).

Excluding the measurement errors because of ECGnon-synchronised CTA, a clinically irrelevant AAA diameter increase after EVAR was observed in eight (72.7\%) of 11 cases. The mean clinically irrelevant diameter increase was $2.04 \pm 0.57 \mathrm{~mm}$. The AAA volume changes were also evaluated in those cases. The mean relative volume increase was $11.50 \pm 8.27 \%$. There was no statistically significant difference between diameter and volumetric AAA 

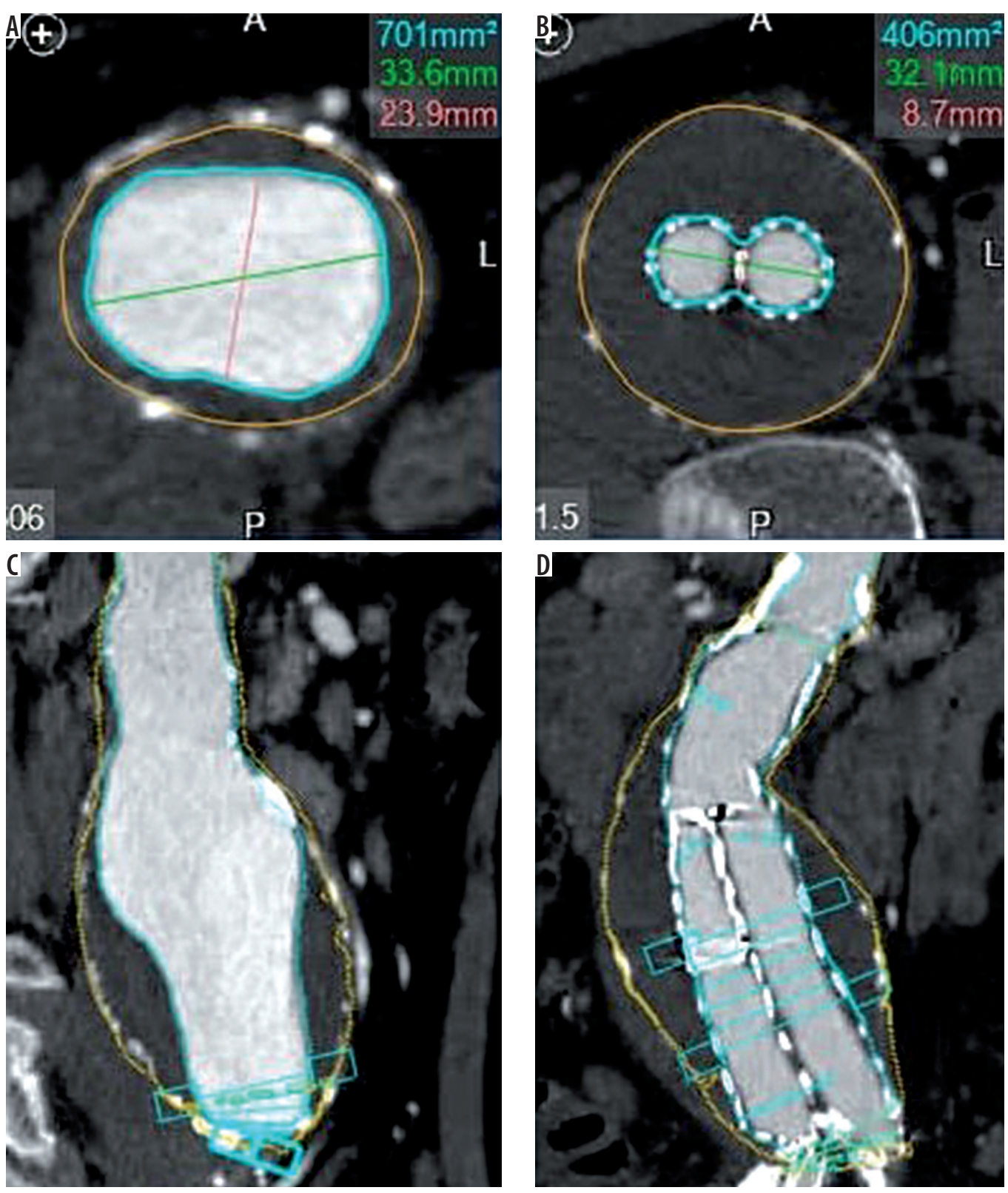

Figure 2. Volumetric abdominal aortic aneurysm measurements

changes in those cases $(p=0.184)$. However, all eight patients were non-smoking men with arterial hypertension and a mean age of $72.25 \pm 8.71$ years.

\section{Association of abdominal aortic aneurysm sac volume and intraluminal thrombus in groups}

The mean volume of ILT of AAA sac was $131.4 \pm 119.6 \mathrm{ml}$ preoperatively $(164.4 \pm 155.5 \mathrm{ml}$ in the growing AAA sac group and $113.0 \pm 73.5 \mathrm{ml}$ in the stable or shrinking AAA sac group). ILT and AAA sac ratios of all cases were calculated for measuring standardisation. The means of ratios were $0.59 \pm 0.17$ and $0.52 \pm 1.8$ in cases in the growing AAA sac group and in the stable or shrinking AAA sac group, respectively. No statistically significant association between post-EVAR growth of AAA sac and preoperative proportion of ILT was found ( $p=0.308)$.

\section{Discussion}

The main findings of our study are as follows: in some cases, the volumetric aneurysm measurement as an additional method to the maximum AAA diameter measurement may be useful in detecting a clinically relevant aneurysm enlargement after EVAR; and the preoperative ILT volume may not predict postoperative AAA sac expansion.

Currently, measurement of maximum aneurysm diameter in post-EVAR surveillance is the most widely accepted method in daily clinical practice. However, the most clinical CTA scans are performed without ECG gating. Therefore, errors may occur in measuring aortic diameter. The main disadvantages of volumetry are that it is time consuming and expensive, post-processing software is necessary, and volumetric segmentation must be accurately performed. In the study we found a moderate 

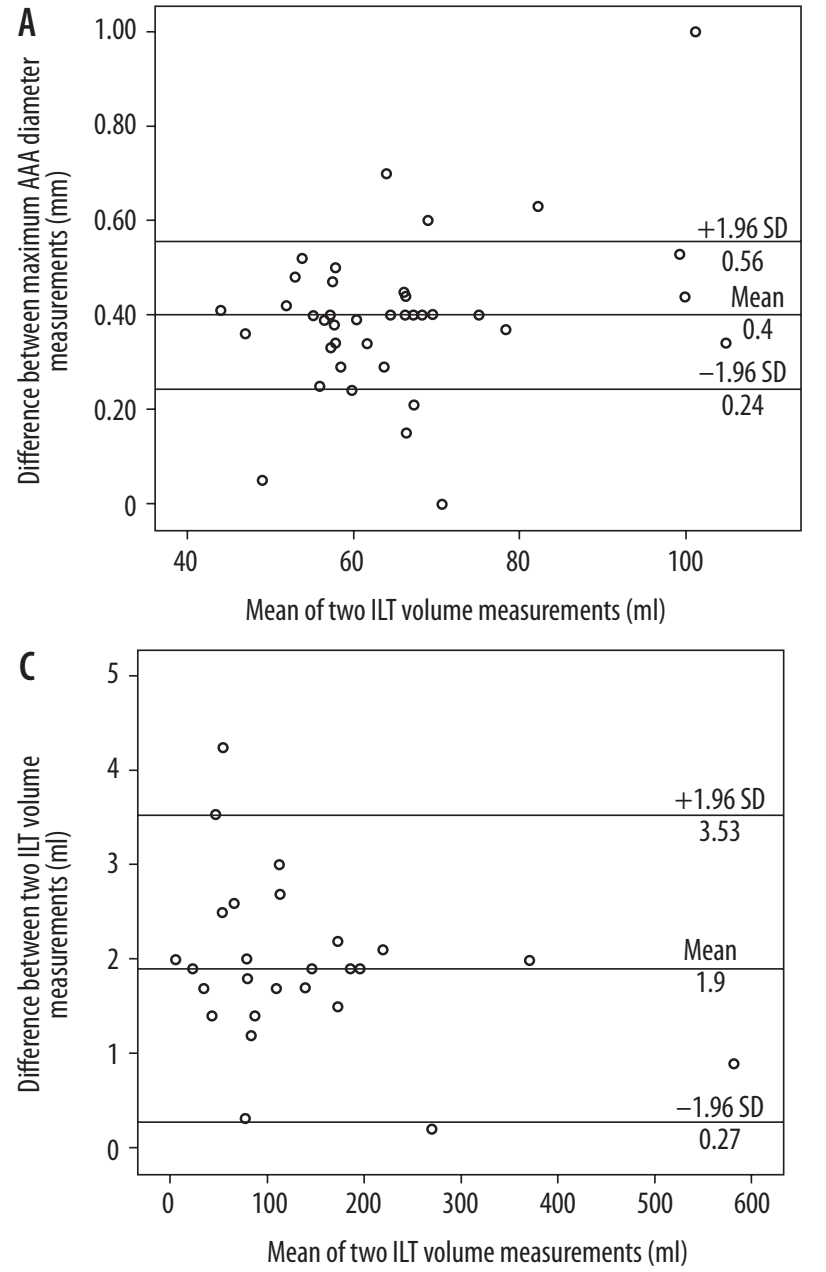

positive linear correlation between the maximum AAA diameter changes and the relative AAA volume changes after EVAR.

Several previous studies revealed a significant discrepancy between volumetric and diametric parameters in the

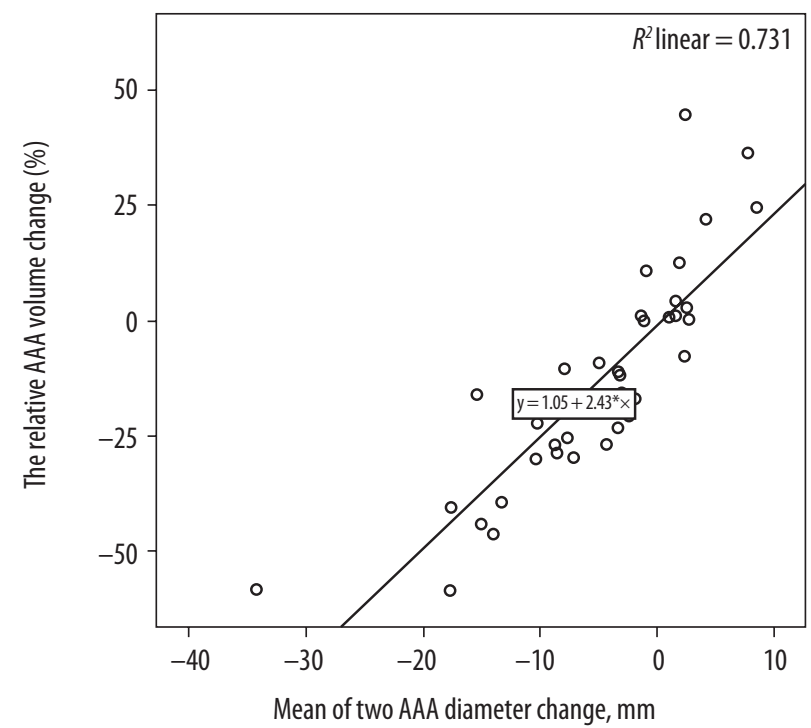

Figure 4. Correlation between the maximum abdominal aortic aneurysm (AAA) diameter changes and the relative AAA volume changes after endovascular aneurysm repair

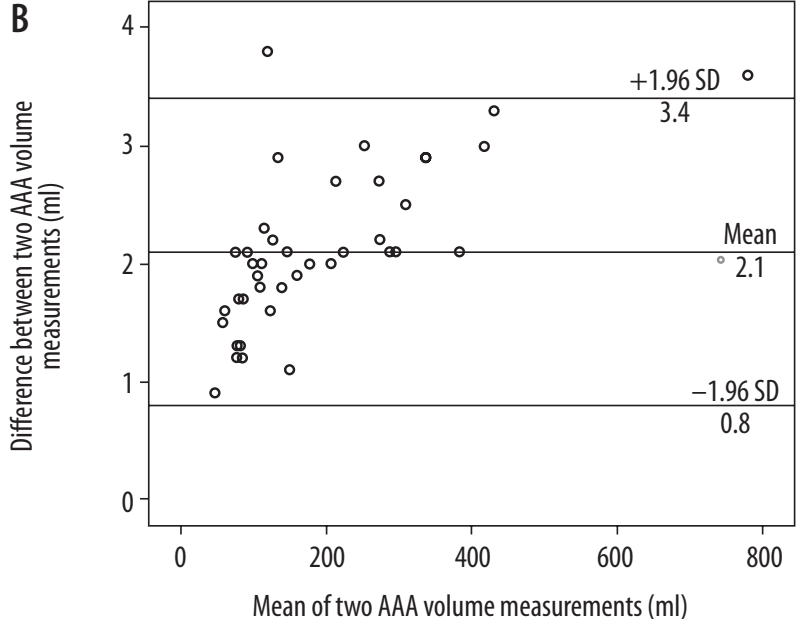

Figure 3. The Bland-Altman plots of maximum abdominal aortic aneurysm diameter (A), volume (B) and intraluminal thrombus volume $(\mathbf{C})$ measurements

AAA - abdominal aortic aneurysm, ILT - intraluminal thrombus, SD - standard deviation

detection of AAA growth [16-20]. The sensitivity of diameter measurements was the lowest and the greatest discrepancy with volumetric parameters noticed at early follow-up (six months or one year) $[18,20]$. The higher sensitivity of volumetry in aneurysm growth detection seems logical because absolute changes of volumetric parameter are greater than diameter. In the study by Parr et al. [16], a $1 \mathrm{~mm}$ increase in orthogonal diameter equated to a $4 \mathrm{ml}$ increase in AAA volume. Moreover, the single diameter measurement does not reflect changes at other sites, for example, lengthening of the aneurysmatic segment [16]. Therefore, volumetric measurements should be considered, especially during early follow-ups. If enlargement is not detected using this method, diametric measurements may be applied for further surveillance and individualisation of its frequency without compromising patient safety.

We analysed the data of patients whose maximum AAA diameter enlargement after EVAR was clinically irrelevant. More than $11 \%$ of AAA volume enlargement was found in those cases. The threshold for clinically relevant volumetric AAA sac expansion after EVAR remains unclear. However, a postoperative aneurysm increase by more than one tenth may be important and may require further investigation to prevent late complications like AAA rupture. In the present study all the patients with irrelevant AAA enlargement after EVAR were non-smok- 
ing men with arterial hypertension and a mean age of 72 years.

No significant association between preoperative volume of ILT and post-EVAR growth of the AAA sac was found in the present study. Some previous studies revealed that the thrombus has a different influence on aneurysm sac behaviour following endovascular treatment, but all these investigations differ considerably. Yeung et al. [13] identified that AAAs with larger preoperative thrombus were associated with failure of aneurysm sac shrinkage in the first 12 months after EVAR in patients who were free from endoleaks. Furthermore, Sirigano et al. [11] reported that a increases of thrombus area by more than $60 \%$ and thrombus volume by more than $59 \%$ were related to higher rates of reintervention. In contrast, other studies associated larger preoperative thrombus with protection from post-EVAR sac enlargement; however, in all of those studies aneurysm growth was related with type II endoleaks [12,21,22]. Hiraoka et al. [22] found that only older age, and not preoperative thrombus burden, was an independent risk factor in the patient group with sac enlargement but without an endoleak. In addition, a study by Rai et al. [23] did not show significant association between preoperative thrombus volume and clinical failure, endoleak, or reintervention after endovascular treatment. Müller-Wille et al. [21] revealed that circumferential thrombus distribution is a protective factor against aneurysm growth following EVAR. It should be noted that it is quite difficult to compare these findings because the results were obtained using different methods of measuring thrombus volume or burden. It is possible that in the case of low preoperative thrombus burden, a lot of free space between the stentgraft and the aneurysm wall remains. It can also be associated with an increased number of patent lumbar arteries because preoperative thrombus is thought to occlude aortic side branches. Therefore, ongoing perfusion and subsequent pressurisation of the aneurysm sac may occur in such conditions [24,25].

As mentioned before, quantitative and qualitative properties of the thrombus may have a significant impact on
AAA remodelling after EVAR. ILT has been demonstrated to be a biologically active structure that contains large numbers of polymorphonuclear leukocytes, high concentrations of cytokines, matrix metalloproteinases (MMPs), serine proteases, myeloperoxidase (MPO), etc. [26-29]. Most of the MMPs and other proteases are secreted by inflammatory cells and platelets entrapped in the thrombus, while MMP-2 is mostly released by vascular smooth muscle cells under hypoxic conditions [27]. Inflammation and enzymatic activity lead to changes in the biochemical state of the AAA wall and its subsequent degradation and weakening.

Further experimental and prospective cohort investigations are necessary to clarify the impact of the thrombus on the aneurysm sac and its behaviour following EVAR.

The main drawback of this study is the modest sample, which may be explained by the relatively short period of surveillance for a small population. The size of the sample may have been impacted by the fact that there are more than five centres in our country, in which EVARs are performed for a total population of 2.5 million. Another significant source of bias is the number of patients lost on follow-up (68 [63.6\%] out of 107).

\section{Conclusions}

Volumetric AAA measurement may be useful as an additional method to diameter measurement after EVAR to identify clinically relevant sac growth. However, further studies are needed to determine in which cases it could be useful. The volume of ILT probably does not significantly affect the growth rate of AAA after EVAR. However, the impact of ILT and volumetric AAA sac changes to aneurysm behaviour after EVAR remains unclear and also requires further investigation.

\section{Disclosure}

The authors declare no conflicts of interest.

\section{References}

1. Rimm EB, Sc D, Chasman DI, et al. New England Journal. N Engl J Med 2012; 367: 1387-96.

2. Powell JT, Sweeting MJ, Ulug P, et al. Meta-analysis of individual-patient data from EVAR-1, DREAM, OVER and ACE trials comparing outcomes of endovascular or open repair for abdominal aortic aneurysm over 5 years. Br J Surg 2017; 104: 166-178.

3. Schlösser FJV, Gusberg RJ, Dardik A, et al. Aneurysm rupture after EVAR: can the ultimate failure be predicted? Eur J Vasc Endovasc Surg 2009; 37: 15-22.

4. Koole D, Moll FL, Buth J, et al. Annual rupture risk of abdominal aortic aneurysm enlargement without detectable endoleak after

endovascular abdominal aortic repair. J Vasc Surg 2011; 54: 16141622.

5. Kirkpatrick VE, Wilson SE, Williams RA, et al. Surveillance computed tomographic arteriogram does not change management before 3 years in patients who have a normal post-EVAR study. Ann Vasc Surg 2014; 28: 831-836.

6. Barnes M, Boult M, Maddern G, et al. A model to predict outcomes for endovascular aneurysm repair using preoperative variables. Eur J Vasc Endovasc Surg 2008; 35: 571-579.

7. Karthikesalingam A, Holt PJ, Vidal-Diez A, et al. Predicting aortic complications after endovascular aneurysm repair. Br J Surg 2013; 100: 1302-1311. 
8. Kaladji A, Daoudal A, Duménil A, et al. Predictive models of complications after endovascular aortic aneurysm repair. Ann Vasc Surg 2017; 40: 19-27.

9. Schnitzbauer M, Güntner O, Wohlgemuth WA, et al. CT after endovascular repair of abdominal aortic aneurysms: diagnostic accuracy of diameter measurements for the detection of aneurysm sac enlargement. J Vasc Interv Radiol 2018; 29: 178-187.e3.

10. Canì A, Cotta E, Recaldini C, et al. Analisi volumetrica della sacca aneurismatica nel follow-up con tomografia computerizzata degli aneurismi dellaorta addominale dopo trattamento endovascolare. Radiol Med (Torino) 2012; 117: 72-84.

11. Sirignano P, Menna D, Capoccia L, et al. Preoperative intrasac thrombus load predicts worse outcome after elective endovascular repair of abdominal aortic aneurysms. J Vasc Interv Radiol 2015; 26: 1431-1436.

12. Sadek M, Dexter DJ, Rockman CB, et al. Preoperative relative abdominal aortic aneurysm thrombus burden predicts endoleak and sac enlargement after endovascular anerysm repair. Ann Vasc Surg 2013; 27: 1036-1041.

13. Yeung JJ, Hernandez-Boussard TM, Song TK, Dalman RL, Lee JT. Preoperative thrombus volume predicts sac regression after endovascular aneurysm repair. J Endovasc Ther Off J Int Soc Endovasc Spec 2009; 16: 380-388.

14. Ahn SS, Rutherford RB, Johnston ICW, et al. Reporting standards for infrarenal endovascular abdominal aortic aneurysm repair. J Vasc Surg 1997; 25: 405-410.

15. Wagner M, Butler C, Rief M, et al. Comparison of non-gated vs. electrocardiogram-gated 64-detector-row computed tomography for integrated electroanatomic mapping in patients undergoing pulmonary vein isolation. Europace 2010; 12: 1090-1097.

16. Parr A, Jayaratne C, Buttner P, Golledge J. Comparison of volume and diameter measurement in assessing small abdominal aortic aneurysm expansion examined using computed tomographic angiography. Eur J Radiol 2011; 79: 42-47.

17. van Keulen JW, van Prehn J, Prokop M, et al. Potential value of aneurysm sac volume measurements in addition to diameter measurements after endovascular aneurysm repair. J Endovasc Ther 2009; 16: 506-513.
18. Fillinger M. Three-dimensional analysis of enlarging aneurysms after endovascular abdominal aortic aneurysm repair in the Gore Excluder Pivotal clinical trial. J Vasc Surg 2006; 43: 888-895.

19. Wever JJ, Blankensteijn JD, Mali WPTM, Eikelboom BC. Maximal aneurysm diameter follow-up is inadequate after endovascular abdominal aortic aneurysm repair. Eur J Vasc Endovasc Surg 2000; 20: 177-182.

20. Bargellini I, Cioni R, Petruzzi P, et al. Endovascular repair of abdominal aortic aneurysms: Analysis of aneurysm volumetric changes at mid-term follow-up. Cardiovasc Intervent Radiol 2005; 28: 426-433.

21. Müller-Wille R, Güntner O, Zeman F, et al. The influence of preoperative aneurysmal thrombus quantity and distribution on the development of type ii endoleaks with aneurysm sac enlargement after EVAR of AAA. Cardiovasc Intervent Radiol 2016; 39: 1099-1109.

22. Hiraoka A, Chikazawa G, Ishida A, et al. Impact of age and intraluminal thrombus volume on abdominal aortic aneurysm sac enlargement after endovascular repair. Ann Vasc Surg 2015; 29: 1440-1446.

23. Rai D, Wisniowski B, Bradshaw B, et al. Abdominal aortic aneurysm calcification and thrombus volume are not associated with outcome following endovascular abdominal aortic aneurysm repair. Eur Radiol 2014; $24:$ 1768-1776.

24. Avgerinos ED, Chaer RA, Makaroun MS. Type II endoleaks. J Vasc Surg 2014; 60: 1386-1391.

25. Brown A, Saggu GK, Bown MJ, Sayers RD, Sidloff DA. Type II endoleaks: challenges and solutions. Vasc Health Risk Manag 2016; 12: 53-63.

26. Folkesson M, Silveira A, Eriksson P, Swedenborg J. Protease activity in the multi-layered intra-luminal thrombus of abdominal aortic aneurysms. Atherosclerosis 2011; 218: 294-299.

27. Koole D, Zandvoort HJA, Schoneveld A, et al. Intraluminal abdominal aortic aneurysm thrombus is associated with disruption of wall integrity. J Vasc Surg 2013; 57: 77-83.

28. Wiernicki I, Stachowska E, Safranow K, et al. Enhanced matrix-degrading proteolytic activity within the thin thrombus-covered wall of human abdominal aortic aneurysms. Atherosclerosis 2010; 212: 161-165.

29. Wilson JS, Virag L, Di Achille P, et al. Biochemomechanics of intraluminal thrombus in abdominal aortic aneurysms. J Biomech Eng 2013; 135: 021011. 